\title{
Norois
}

Environnement, aménagement, société

$232 \mid 2014$

Modes de vie, modes d'habiter des aînés entre inclusion et exclusion

\section{L'inclusion à la ville des personnes âgées : entre déprise et citadinité}

L'exemple des promenades balnéaires

Inclusion of older people in the city: "déprise" and urbanity. The example of seaside promenades

Mathilde Bigo et Sandrine Depeau

\section{(2) OpenEdition}

Journals

Édition électronique

URL : https://journals.openedition.org/norois/5135

DOI : 10.4000/norois.5135

ISSN : 1760-8546

Éditeur

Presses universitaires de Rennes

Édition imprimée

Date de publication : 30 octobre 2014

Pagination : 11-22

ISBN : 978-2-7535-3974-7

ISSN : 0029-182X

Référence électronique

Mathilde Bigo et Sandrine Depeau, «L'inclusion à la ville des personnes âgées : entre déprise et citadinité », Norois [En ligne], 232 | 2014, mis en ligne le 30 novembre 2016, consulté le 13 janvier 2022. URL : http://journals.openedition.org/norois/5135; DOI : https://doi.org/10.4000/norois.5135

(ㄷ) Tous droits réservés 


Presses
Universitaires
de Rennes
www.pur-editions.fr
Revue en ligne : http://norois.revues.org

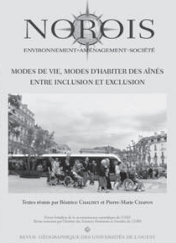

\title{
L'inclusion à la ville des personnes âgées : entre déprise et citadinité L'exemple des promenades balnéaires
}

\author{
Inclusion of Older People in the City: "Déprise" and Urbanity \\ The Example of Seaside Promenades
}

Mathilde Bigo*, Sandrine Depeau

*Auteur correspondant: 0299142098

ESO-Rennes - UMR 6590 CNRS, Université Rennes 2, MRSS, place du Recteur-Henry-Le-Moal - 35043 RENNES

cedex (mathilde.bigo@univ-rennes2.fr) (sandrine.depeau@univ-rennes2.fr)

Résumé : Vieillir chez soi représente un désir fort des personnes âgées et une priorité des politiques publiques qui exige d'appréhender les modes d'habiter dans la diversité des lieux de vie des personnes âgées. La question posée dans cet article revient alors à interroger la notion d'inclusion à partir du rapport à l'espace urbain. Pour comprendre les modes d'inclusion à la ville des personnes âgées deux notions articulées sont discutées ici : la déprise, relative au vieillissement, et la citadinité, pour ce qui est du rapport à la ville, et plus précisément l'espace urbain littoral de la station balnéaire. L’objectif de l'article est de poser une exploration théorique qui vise à conceptualiser l'inclusion des personnes âgées à la ville en considérant le processus de vieillissement à travers les transactions individus-ville.

\begin{abstract}
Growing old at home is a strong wish of the elderly and a priority for public policy that requires an understanding of how the elderly dwell in different types of places. Therefore, we aim to examine the notion of inclusion regarding the relationship to public space. To understand the modes of inclusion of the elderly in the city, two articulated notions are explored and discussed here: "déprise" (spatial withdrawal from public space, in relation to ageing) and "urbanity" (in terms of relationships to the city and more specifically to the coastal urban space of the seaside resort). The aim of the article is to conduct a theoretical study to consider the social inclusion of the elderly in the city by focusing on the process of ageing in the interactions between people and the city.
\end{abstract}

Mots clés : citadinité - inclusion - déprise - vieillissement - balnéaire - espace public urbain

Keywords: urbanity - inclusion - abandonment - ageing - seaside - urban public space

\section{INTRODUCTION}

La notion de «vieillir chez soi » constitue aujourd'hui une des priorités des politiques publiques. En effet, en même temps qu'elle correspond à un désir de plus en plus fort des personnes âgées, elle oblige à repenser autant les modes d'or- ganisation des services à la personne que les modes d'habiter de façon de plus générale. Aussi, le « chez soi » doit être entendu dans une acception large qui le définit comme un système d'espaces comprenant le logement mais aussi les espaces collectifs et les espaces publics du contexte de vie des personnes. 
En l'occurrence ici, des contextes de vie urbains. Autrement dit, «vieillir chez soi » ne se limite pas à la coquille de l'intime que forme le logement mais comprend également la diversité des espaces publics auxquels les personnes âgées doivent pouvoir avoir accès pour garantir certaines formes d'autonomie et surtout amortir les effets psychologiques et sociaux que peut entraîner le processus de vieillissement.

La place des personnes âgées dans la cité constitue un enjeu important autant pour les politiques de santé publique que d'aménagement du territoire. L'espace de la ville dans sa matérialité peut être un obstacle ou au contraire une condition d'accès au monde social, et inversement, l'organisation sociale peut être aussi une condition des pratiques spatiales. L'inclusion à la ville implique donc de considérer la ville comme une entité aux dimensions matérielles et sociales.

Dans cet article, il s'agit de montrer comment l'inclusion des personnes âgées doit être entendue à partir de relations qu'elles ont avec la ville, entité spatiale et sociale, ainsi que du processus de vieillissement qui peut mettre à mal l'inclusion de la personne.

La question posée dans cet article revient alors à interroger l'inclusion des personnes âgées dans l'espace public en travaillant deux notions : la citadinité et la déprise.

Dans cette perspective, l'article s'organise en trois parties. Nous reviendrons dans un premier temps sur les enjeux de penser la ville pour les personnes âgées. Il s'agira d'aborder l'espace de la ville, non seulement dans ses caractéristiques physiques, et en particulier à partir de la notion de walkability, mais également dans le rapport à l'accompagnant et aux autres usagers de l'espace public. Puis, nous détaillerons les deux notions clés de notre propos : la citadinité et la déprise, afin de saisir l'articulation qui se joue pour interroger l'inclusion des personnes âgées en ville. Enfin, nous interrogerons le rôle de certains espaces de la ville, comme cadre et support à l'inclusion des personnes âgées, à savoir les promenades balnéaires sur le littoral breton. Particulièrement touchées par le vieillissement de la population, les communes littorales bretonnes font face à de nouveaux enjeux sociaux et d'aménagement en termes d'inclusion des personnes vieillissantes dans la ville. Les promenades balnéaires seront alors interrogées sur leur propension à être un espace de citadinité, ressource pour l'inclusion des personnes âgées.

\section{Penser la ville POUR LES PERSONNES ÂGÉES}

On peut déplorer que les études en gérontologie se soient focalisées principalement sur le maintien «à domicile », et donc sur le logement (Clément, 1994; Clément et al., 1998). Le recensement des attentes et besoins de la population vieillissante en ville permet d'insister sur la nécessité de penser le vieillissement au-delà de l'espace du logement, dans l'espace public, à l'appui de l'expression « home beyond the house » (Cloutier-Fisher et Harvey, 2009).

\section{Quelle forme de ville favorable aux personnes âgées?}

Si l'avancée en âge peut expliquer une part de la réduction des usages de l'espace, corrélativement à une diminution des capacités, il est généralement admis que les aménagements des espaces urbains sont peu favorables aux personnes âgées (Clément, Mantovani, et Membrado, 1996; Mollenkopf et al., 1997 ; Membrado, 1998; Pennec, 2005; Lord et Després, 2011).

Les recherches sur le vieillissement en ville s'accordent sur les particularités des espaces, relevées à partir des besoins des personnes âgées, concernant un meilleur accès aux services, un cadre de vie bien entretenu, et le besoin de sécurisation et de confort. Ces recherches portent généralement sur l'échelle piétonne de la ville, justifiée par le fait que la marche est privilégiée par les seniors, autant comme activité physique que comme moyen de transport (Ridolfi et Dumont, 2006; Borst et al., 2008; KT-EQUAL, 2010). L'enjeu est alors de penser un espace inclusif, ou inclusive design (KT-EQUAL, 2010) pour favoriser la marche à pied, et ainsi promouvoir la santé (Michael, Green et Farquhar, 2006; Borst et al., 2008; KT-EQUAL, 2010 ; Touboul et al., 2011). Cette façon de penser la ville pour les personnes âgées rejoint de très près les principes de la walkability définie par des indicateurs de sécurisation, d'esthétisme, de nature, d'aménités piétonnes, de connectivités des rues (Saelens, Sallis, et Frank 2003; Ramirez et al., 2006; Brown et al., 2007). Nous nous attarderons ici sur trois d'entre eux : la sécurisation, les aménités piétonnes, l'esthétisme, pour comprendre comment ils sont abordés dans les recherches sur la ville et les personnes âgées. 
La sécurisation des personnes âgées dans les espaces publics fait l'objet de rapports établissant le bilan des principales difficultés rencontrées par les personnes âgées en ville du fait d'un déclin moteur et cognitif (Heam et Dejeammes, 2000; Dommes, Cavallo, et Boustelitane, 2008). Certains auteurs (Clément, 1994 ; Pennec, 2005) précisent qu'audelà de la sécurité des piétons âgés vis-à-vis de la circulation automobile, les risques liés à l'utilisation des transports en commun par les personnes âgées ne sont pas à négliger, à cause de l'accès aux véhicules qui leur est malaisé. Elles peuvent donc, en retour, être considérées comme des sujets à risque du fait d'une moindre fluidité dans les transports. Ce qui pousse à préconiser aux municipalités d'optimiser les «déplacements slow » (Ridolfi et Dumont, 2006).

Les principaux éléments de confort urbain repérés à ce sujet sont l'éclairage, la largeur des trottoirs, la piétonisation et le mobilier (Carmona, 1985; Masboungi et Bourdin, 2004). Les infrastructures piétonnes, telles que les trottoirs, les plus larges possible, les passages cloutés, et, mieux encore, les zones dites piétonnes, sont des caractéristiques favorables au déplacement à pied des personnes âgées (Mollenkopf et al., 1997 ; Michael, Green, et Farquhar, 2006; Ridolfi et Dumont, 2006; Borst et al., 2008). En somme, avec l'avancée en âge, les obstacles aux déplacements sont généralement plus importants, et les personnes âgées doivent faire face à « la brutalité de l'espace construit » (Olivera et Abellan, 1994). Le manque de sécurisation et de confort peut être rédhibitoire dans leur pratique de la ville : le moindre relief, la moindre aspérité, le moindre obstacle, tout ce qui peut générer de la gêne pour le corps et un sentiment d'insécurité devient une contrainte pour les pratiques spatiales. L'accessibilité à la ville pour les personnes âgées en situation de handicap a fait l'objet d'une mesure, au regard de la pénibilité des déplacements. Cette mesure prend en compte la forme de la voirie : largeur et abaissement des trottoirs, nature du sol, mobilier urbain (Chaudet, 2004).

L'esthétique des espaces publics participe elle aussi de l'attractivité des lieux. Les personnes âgées sont demandeuses d'un espace public propre, bien entretenu, joli (Ridolfi et Dumont, 2006; Bésingrand et Soumagne, 2006), avec la présence de végétation, ou encore le témoignage de marques architecturales historiques attrayantes. À l'inverse, la densité de l'habitat, les grands immeubles, les détritus sur la chaussée sont des aspects de la ville qui n'encouragent pas les déplacements à pied des personnes âgées (Michael, Green, Farquhar, 2006; Borst et al., 2008).

On peut se demander, au vu de ces caractéristiques de commodité de déplacements pour les personnes âgées, si, au final, penser la ville pour le vieillissement ne serait pas aussi une façon de penser le bien-être urbain de façon plus générale (Pihet, 2006) ? De la même façon que poser les critères d'une ville accessible aux personnes porteuses de handicap (Bodin, 2002) ou aux enfants (Depeau, 2003) revient à penser les enjeux d'accessibilité à la ville pour toutes et tous, quelles que soient ses capacités corporelles (Cluzet, 2003).

\section{Quels rapports aux autres dans l'espace public pour les personnes âgées?}

Parallèlement à la dimension matérielle de l'espace urbain, la dimension sociale est nécessaire pour comprendre les pratiques des personnes âgées en ville. En effet, les pratiques hors du domicile peuvent être assimilées à des transactions ${ }^{1}$ individuespaces qui impliquent les caractéristiques personnelles et les caractéristiques socio-spatiales des lieux parcourus.

Le rapport aux autres dans l'espace public se comprend autant à travers les aides au déplacement, le réseau social, que dans l'expérience de la confrontation sociale. Certaines études relatives à la vieillesse dans les espaces urbains s'appuient justement sur l'opposition entre, la confrontation avec les autres, et l'entre soi, pour aborder les pratiques des personnes âgées. Par exemple, pratiquer la ville en s'alignant sur le temps des actifs, quand il y a foule, «permet d'être semblable au monde alentour » (Pennec, 2006, p. 54) et d'éprouver un sentiment de faire partie du monde (Lord, Després, et Ramadier, 2011), en même temps que « voir sans être trop vu devient l'objectif de cette présence anonyme pour s'assurer d'être en prise avec le monde d'aujourd'hui » (Pennec, 2006, p. 54). À l'opposé, la peur de la confrontation avec

\footnotetext{
1. L'approche transactionnelle en psychologie environnementale permet de mettre l'accent sur la relation entre l'individu et l'espace pratiqué. Elle «donne donc toute sa place au contexte de l'échange, de la relation, du lien, et donc à l'espace et aux lieux dans lesquels ces négociations s'opèrent » (Séchet, Garat, et Zeneidi, 2008, p. 19)
} 
les jeunes dans l'espace public et le sentiment d'être dépassé (Caradec, 2004), conduisent à élaborer des stratégies pour éviter les heures d'affluence, se protéger des autres, ce qui favorise l'entre soi (Clément, Mantovani, et Membrado, 1996; Clément, Mantovani, et Membrado, 1998; Guillemot et Soumagne, 2007). En somme, la confrontation dans l'espace public est un élément de la vie sociale urbaine qui influence largement les pratiques de l'espace par les personnes âgées, que cette confrontation soit recherchée ou fuie. Certaines études font l'état de l'importance du réseau social dans les pratiques des personnes âgées en ville (Mollenkopf et al., 1997; Pennec, 2005 ; 2006). S'il est établi que le conjoint est en priorité celui qui structure les déplacements hors du domicile, il faut préciser que les enfants, les petits-enfants, les amis, les voisins, la fratrie, mais aussi les professionnels des services sociaux, sont autant de liens sans lesquels la sortie du domicile n'aurait parfois pas lieu; des liens qui sont différenciés selon les ressources familiales et amicales de la personne âgée. Le voisinage d'ailleurs pourrait être un des derniers liens affectés par l'avancée en âge et permettrait d'avertir la famille ou un professionnel en cas de problème (Membrado, 2003; Drulhe et al., 2008).

L'intérêt d'une approche du vieillir chez soi qui s'étend aux espaces pratiqués de la ville permet de bien comprendre ce qui se joue pour l'individu vieillissant dans son quotidien. Elle interroge la citadinité, autrement dit le rapport à la ville et donc au monde. Il y a alors nécessité à mettre en relation le vieillissement et les caractéristiques de la ville, afin de mesurer la « congruence » physique et sociale et donc, selon Alves (2011), le caractère habitable (la «liveability») de l'environnement quotidien des personnes âgées dans l'analyse de la vie quotidienne et des pratiques courantes.

\section{Penser l'inclusion DES PERSONNES ÂGÉES À LA VILLE}

Si l'on veut mesurer la congruence de l'espace de vie des personnes âgées, il semble nécessaire de s'intéresser à la diversité des rapports aux espaces. Pour cela, la citadinité permet de rendre compte de la relation entre l'individu et la ville en tenant compte autant des caractéristiques personnelles de l'individu (ici il s'agit des caractéristiques du vieillissement tels le changement de rôle social et l'amoin- drissement physique) que des caractéristiques de la ville (comprise comme entité sociale, matérielle et symbolique). En la combinant à la notion de déprise, propre au processus de vieillissement, nous montrons comment il est possible d'opérationnaliser conjointement ces deux notions pour appréhender l'inclusion des personnes âgées en ville.

\section{La citadinité : une relation entre l'individu et la ville}

L'étude de la citadinité est celle du lien des individus à la ville, et des individus entre eux par le biais des pratiques spatiales. Elle impose de penser la relation entre l'individu et la ville de façon dynamique, comme un processus de co-construction (Berry-Chikhaoui, 2009) qui se nourrit des discours et des pratiques individuelles et collectives, relatives aux significations que les individus attribuent aux lieux, à la représentation qu'ils se font de la ville (Berry-Chikhaoui et Deboulet, 2000; GervaisLambony, 2001 ; Lussault, 2003). Ce « système de signes » produit à partir des pratiques et représentations du citadin et fournissant les preuves de la relation que l'individu entretient avec la ville (Lussault, 2003) révèle la dimension subjective de la citadinité (Gervais-Lambony, 2001).

La notion de citadinité permet d'étudier la ville vécue "de l'intérieur» par les habitants. Certains auteurs parlent d'une approche «par le bas » (BerryChikhaoui, 2009). Celle-ci tient compte des « compétences » des citadins définies, au sens de Giddens (1987), à la fois par la connaissance et par la capacité à utiliser sa connaissance. Ce qui permet de considérer l'individu comme un acteur ayant à la fois des compétences énonciatrices et une approche réflexive sur sa pratique de la ville (Lussault, 2003). La compétence citadine serait celle de faire lien avec les autres, de trouver sa place parmi les autres.

Parce que la définition s'appuie sur la relation entre individu et objet urbain, la citadinité se rapproche des notions d'habiter et de territorialité et met en jeu les questions de sentiment d'appartenance, d'appropriation, d'intégration, d'identité. La citadinité pourrait donc se définir comme une relation dynamique entre individu et ville produite par des interactions sociales. Celle-ci limitent et déterminent les comportements communément acceptés et reconnus par les usagers de l'espace public selon 
les lieux de la ville. De cette relation découlerait une inclusion des individus dans la ville, et une forme d'incorporation de la ville dans les pratiques individuelles. Dans le cas des personnes âgées, sortir pour faire ses courses de façon autonome par exemple, nécessite un environnement urbain et social « affordant » (adapté aux attentes des individus concernés) qui permet à la personne âgée d'être incluse dans la société en pratiquant la ville au même titre que l'ensemble des citadins. Autrement dit, comme le rappelle ici Gervais-Lambony (2001) qui cite les propos de Jaillet (1999), la citadinité est fondée sur un « vivre ensemble », qui produit «non pas de la socialité seulement, mais une société, c'est-à-dire une capacité collective à faire tenir ensemble des groupes fortement différenciés » (p. 158).

Pour mesurer et qualifier la citadinité, certains auteurs parlent de degré de citadinité (Gervais Lambony, 2001). On préférera le point de vue de Berry-Chikhaoui qui troque les degrés de citadinité contre des citadinités plurielles (Berry-Chikhaoui, 2009). Chacune des citadinités est unique. De fait, afin de rendre opérationnelle l'analyse de la citadinité des personnes âgées, nous avons défini trois dimensions de la citadinité.

\section{Première dimension : le rapport à l'espace urbain}

Cette dimension regroupe des indicateurs de la compétence à faire ville avec les autres. Elle sert à positionner l'individu par rapport à ce qu'est la ville dans ses dimensions sociales, matérielles et symboliques. Autrement dit, elle met l'individu face aux autres et à l'espace construit, deux éléments qui, on l'a vu, ont un poids important lors du vieillissement en ville. Ainsi, l'imprévu des rencontres, l'accès à la centralité, la flânerie et l'utilisation des services urbains, sont des indicateurs du rapport à l'espace urbain. Ils se doivent d'être considérés sous le filtre des temporalités sociales qui rythment cet espace afin de bien distinguer les expériences de l'entre soi, de celles de la confrontation sociale.

\section{Deuxième dimension : le rapport aux pairs}

Le rapport aux pairs signifie ici les liens sociaux qui font exister l'individu dans un groupe social, qui lui donnent un rôle au sein du groupe. Le choix d'appréhender les liens sociaux entretenus par l'individu relève de l'hypothèse que les sociabilités sont parties intégrantes du processus d'appropriation de l'espace de la ville, contribuant à un « ancrage spatial»(Di Méo). Il peut s'agir aussi bien d'amis, de famille, que des individus en lien avec les services sociaux d'aide à la personne, autrement dit «the most important person " pour reprendre Mollenkopf (Mollenkopf et al., 1997). L'accompagnement lors des sorties hors du domicile, ainsi que les rencontres de connaissances dans l'espace public, sont les indicateurs de cette seconde dimension.

\section{Troisième dimension : le rapport au passé}

Cette dernière dimension de la citadinité inscrit l'individu par rapport au passé de la ville et de soi dans la ville, afin de rendre compte de la connaissance de la ville dans un temps plus ou moins long Elle peut modérer d'une certaine façon les deux premières dimensions qui sont effectives dans le présent. C'est-à-dire qu'elle met en lumière des pratiques révolues qui ont fondé la relation à la ville et qui continuent, encore aujourd'hui, à fournir des prises pour l'individu qui peuvent de fait se raccrocher à sa mémoire et ses souvenirs pour se sentir « de » la ville. Les deux indicateurs de cette dimension sont donc la capacité à se replacer, soi, dans le passé de la ville, ainsi que la conscientisation des évolutions dans et de la ville.

Les dimensions de la citadinité sont plus ou moins affirmées car tout individu compose la sienne selon son expérience dans les espaces urbains, dans le rapport à l'autre et selon ses propres capacités corporelles. Les trois cas empiriques présentés ci-après pour illustrer le cadre théorique de la citadinité permettent de comprendre ce que serait celle-ci selon une dimension privilégiée dans leurs pratiques. Il s'agit d'exemples extrêmes car une seule des dimensions y est développée. Le premier cas concerne les individus pour qui le rapport à l'espace urbain est le fondement de leur citadinité : envie de flâner, de voir des gens passer, de se confronter à l'imprévu, à la densité d'un centre-ville. Le deuxième cas est celui dont le rapport aux pairs fait la relation à la ville. C'est parce qu'ils sont en ville avec leurs proches ou parce qu'ils retrouvent des connaissances, que ces individus entretiennent leur citadinité. Le groupe soutient la pratique de la ville. 
Enfin, le troisième cas, pour les individus qui ont un fort rapport au passé, la ville existe surtout dans leurs souvenirs. Ce sont les pratiques passées, la mémoire individuelle ou collective des lieux pratiqués qui façonnent aujourd'hui leur citadinité. Par le biais du passé ils gardent prise sur la ville.

Par ailleurs, les concepts d'invention, d'adaptation, de détournement, de substitution (Berry-Chikhaoui et Deboulet, 2000 ; Bouillon et al., 2007) sont intéressants à prendre en compte car ils insistent sur la singularité de la citadinité pour chaque individu et permettent d'aller au-delà d'une citadinité conventionnelle, en intégrant des caractéristiques personnelles des individus et des singularités spatiales qui structurent cette relation. Dans le contexte du vieillissement en ville, il s'agit d'appréhender la citadinité des personnes âgées en rendant compte de ces réajustements, de ces substitutions nécessaires dans les pratiques de la ville lorsque la vieillesse impose une réorganisation de la vie. La notion de déprise, qui caractérise le processus de vieillissement, va permettre d'enrichir celle de citadinité pour appréhender l'inclusion des personnes âgées en ville. Selon ses auteurs, la déprise aide à ne pas conclure trop hâtivement sur le repli sur l'espace du domicile, car ce n'est pas un territoire qui est abandonné, mais des « bouts de ville » qui sont choisis, tandis que d'autres perdent de la valeur (Clément, Mantovani et Membrado, 1998).

\section{Le processus de vieillissement à travers la notion de déprise}

Formalisée pour la première fois en 1988 (Barthe, Drulhe et Clément, 1988), suite à la théorie du désengagement élaborée dans les années 1960 aux Etats-Unis, avant d'être critiquée pour son caractère trop normatif, la déprise a permis de renouveler l'approche du vieillissement. Tout en reconnaissant un certain "relâchement » des personnes âgées, ses auteurs estiment qu'il n'est ni total, ni linéaire, ni homogène. Sous l'effet de l'amoindrissement de l'énergie vitale, on assiste à un abandon de certaines activités, à une baisse des relations sociales et au désir de se mettre en retrait. Ce qui nécessite une réorganisation de la vie et des stratégies de substitution des activités. «Il s'agit de "ne plus avoir la prise" sur certaines choses, situations ou relations, sans exclure qu'elles [les personnes âgées] continuent à "avoir la main haute" sur d'autres » (Barthe, Drulhe, et Clément, 1988, p. 41).

Ainsi, jusque dans des travaux récents, analyser le vieillissement dans une logique de dynamique des pratiques, et non dans une logique d'abandon (Hénaff-Pineau, 2009) demeure l'essence de ce concept de déprise, bien qu'il ait fait l'objet de plusieurs remaniements et précisions.

La déprise est aussi le produit d'interactions interindividuelles : des relations avec les proches qui peuvent dissuader la personne âgée de continuer à exercer certaines activités, ou bien des conduites induites par certaines relations dans l'espace public, comme celles avec les plus jeunes dans l'espace public qui conduisent à des stratégies d'évitement (Clément, Mantovani et Membrado, 1996). D'ailleurs, ce qui différencie la déprise du désengagement serait de rendre compte des changements qui affectent le rapport aux autres dans la vie de l’individu (Clément, 2003).

Quatre déclencheurs de la déprise sont posés et repris dans les différents travaux de Caradec (2004; 2005 ; 2009; 2012) : la fatigue ou le manque d'envie, les interactions avec autrui, les difficultés physiques, la raréfaction des opportunités d'engagement. Si l'équipe de Clément (Clément, Mantovani et Membrado, 1996) présentait déjà la déprise comme un phénomène social, produit de l'interaction avec les autres, notamment dans l'espace public urbain où l'évitement de l'autre (notamment les jeunes) peut amener à des logiques d'exclusion et d'autoexclusion, Caradec (2005) insiste sur le besoin de ne pas réduire le vieillissement à un phénomène psychologique voire physiologique.

\section{Citadinité et déprise : appréhender l'inclusion}

De fait, déprise et citadinité sont deux notions qui peuvent être articulées pour mieux comprendre le rapport aux espaces et la notion d'inclusion. La notion de déprise place la personne âgée dans une perspective dynamique (soit "proactive and developmental" dans le texte) (Lord, Després et Ramadier, 2011). Elle rend compte des changements avec l'avancée en âge dans l'appréciation des lieux et des réajustements dans les besoins et aspirations. Cette notion pose la question des pratiques renouvelées de la ville qui émergent suite à ces nouvelles aspirations. 
La citadinité, quant à elle, interroge, comme on l'a vu, la relation aux autres, aux caractéristiques de l'organisation urbaine, mais aussi l'appropriation des lieux et le sentiment d'appartenance. Elle place l'individu comme acteur de la ville, quel que soit son statut social, et rend compte de son rapport au monde. Si l'on reprend Berry-Chikhaoui et Deboulet (2000), la citadinité repose sur des compétences qui permettraient de faire preuve d'invention, de création, pour faire face à des changements dans l'organisation de l'espace, à des migrations d'un espace à l'autre. Ici, on se pose la question en termes non seulement de confrontation à l'espace qui évolue sans cesse, mais aussi, et surtout, au regard des changements (social, corporel) relatifs à la vieillesse qui fondent un rapport singulier à l'espace. Ainsi, c'est moins le réel de l'espace qui change que la transaction entre individu et ville et donc les modalités de la citadinité qui sont sans cesse à renouveler. L'articulation des notions de déprise et de citadinité pour appréhender l'inclusion des personnes âgées peut être schématisée de la façon suivante (figure 1). Les trois éléments qui structurent cette problématique sont la déprise, la citadinité, l’inclusion. La déprise, par le biais des déclencheurs détaillés plus haut, façonne la citadinité. Celle-ci s'observe entre autres dans les pratiques en ville, les interactions avec les autres et l'appropriation de l'espace qui en découle. Selon le degré de déprise généré par les déclencheurs, mais aussi selon le type de déclencheur engagé, la forme de la citadinité sera différente. Ainsi, avec le temps et le processus de vieillissement qui fait son œuvre, la relation des individus à la ville (ou citadinité) évolue. Cette dernière définit à son tour l'inclusion des personnes âgées, qui, selon son intensité, peut freiner ou accélérer le processus de déprise. Ainsi, lorsque la citadinité est forte $(+)$, l'inclusion l'est aussi $(+)$, ce qui permet d'amortir la déprise (-). À l'inverse, lorsque la citadinité est faible (-), il en est de même pour l'inclusion (-) qui risque de renforcer ou d'accélérer le processus de déprise $(+)$.

Citadinité et déprise sont deux notions qui covarient et permettent de mieux investiguer le rapport à l'autre. La première rend compte du rapport à la ville quand la seconde permet de saisir le cadre de l'expérience du vieillissement. L'une, comme l'autre, doit être appréhendée en prenant en considération les contextes socio-spatiaux. En effet, autant les caractéristiques sociales que les caractéristiques matérielles contribuent à mettre à l'épreuve la notion de déprise qui souvent suppose un environnement matériel défavorable. Comme le précise Caradec (2004), le déclencheur « difficultés physiques » est à considérer essentiellement à partir de l'environnement, au sens large de ce qui

Figure 1 : Articulation des notions de déprise, citadinité, inclusion Articulation for "deprise", urbanity, inclusion

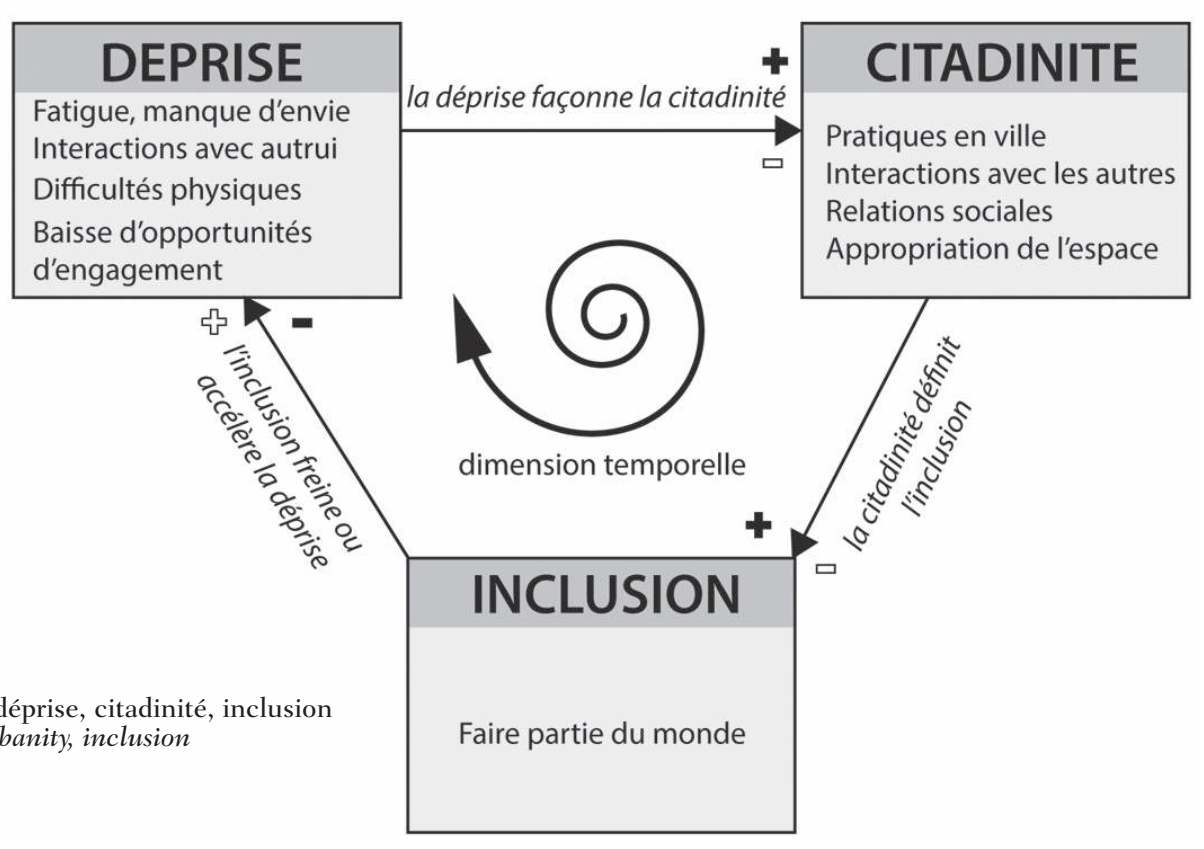


entoure l'individu, car il faut mettre l'accent sur l'inadéquation entre individu et espace, plutôt que sur les problèmes de santé que rencontre l'individu. Ainsi, l'amoindrissement corporel qui demeure une donnée observable fréquente avec l'avancée en âge, n'entraîne pas forcément de contraintes dans la pratique de la ville si celle-ci est accessible. A contrario, si l'espace urbain dans ses formes architecturales et urbanistiques est jalonné d'obstacles qui gênent l'accessibilité au milieu physique, il peut être un frein pour l'accès à la vie sociale (Oliveira et Abellan, 1994). Mais on peut aussi se demander si la déprise ne modifierait pas l'appréciation de la présence de l'autre (Hartig et Staats, 2006).

La réduction de l'étendue des espaces parcourus (Barthe, Drulhe et Clément, 1988), la préservation d'espaces essentiels (Clément, Mantovani et Membrado, 1996), mais aussi le maintien d'activités et de relations sociales, considérées comme moins fatigantes au moment de la baisse des capacités seront ici la traduction de pratiques citadines. Lesquelles ont pour cadre l'espace public urbain, haut lieu du rapport à l'autre, reflet des rapports de hiérarchie sociale, producteurs de normes, espace par excellence de mise à l'épreuve de l'individu dans ses rapports sociaux et dans son rapport aux espaces.

L'espace public urbain peut néanmoins prendre des formes variables. Il façonne la citadinité des individus âgés de façons différentes selon la matérialité et le rapport à l'autre qu'il propose. Espaces urbains singuliers, les promenades balnéaires ont été choisies comme espaces laboratoires pour appréhender la citadinité des personnes âgées, et donc leur inclusion parmi les autres. La suite de l'article veut mettre en évidence les caractéristiques de ces espaces aménagés en bord de mer afin de questionner leur capacité à « inclure » les personnes âgées.

\section{LA PROMENADE BALNÉAIRE : « ESPACE LABORATOIRE » POUR APPRÉHENDER L'INCLUSION DES PERSONNES ÂGÉES}

\section{Un espace de la ville singulier}

Les promenades balnéaires ont été construites dans les communes littorales de villégiature dès le XIX ${ }^{\mathrm{e}}$ siècle, pour se protéger des marées (Delignon, 1998). Ces espaces ont constitué au fil des siècles des lieux de déambulation, d'exposition de soi et des hiérarchies sociales, véritables cours de la station balnéaire (Barbedor et al., 2001 ; Rouillard, 1984).

Aujourd'hui, les promenades balnéaires ont conservé leur fonction sociale, elles sont le lieu de sociabilités de la station littorale (Duhamel et Violier, 2009). Architecture balnéaire oblige, la promenade tend à produire des formes particulières d'interactions sociales. La mise en scène théâtrale, chorégraphiée par les usagers qui défilent, paradent, ou du moins qui se montrent, exacerbe le rapport à l'autre, aussi bien pour ceux qui « observent » que pour ceux qui sont observés. La promenade de bord de mer semble en effet être un espace où le rapport à l'autre est mis en exergue tant par la forme singulière de la promenade, que par sa place dans la ville qui fait de lui un espace central où la confrontation à l'autre est quasi permanente.

Dans les années 1970, de nombreuses villes tentent des expériences d'aménagement visant à piétonniser les centres urbains afin de réduire la circulation automobile (Von Der Mühll, 2004; Dumont et Von Der Mühll, 2006). Dans les stations balnéaires, après des décennies du " tout automobile ", les années 1970 sont également celles des premières tentatives pour revenir aux débuts de l'édification des promenades, avec fleurissement et voie piétonne (Debié, 1993). Les promenades bénéficient des critères d'exigence en matière de voirie. Espaces piétonniers agréables et sûrs à pratiquer, elles répondent aux besoins de confort et de sécurisation (du fait de leur séparation avec la circulation automobile), d'apport de vie sociale et d'animation culturelle par le biais des commerces (Fischer, 1981) ainsi qu'au souci d'esthétique paysagère (Fougnie, 2008). Si la mise en place de zones piétonnes a pour objectif la sécurisation des déplacements, elle permet aussi de façon plus générale de mieux apprécier la ville en s'isolant de ses désagréments (Cadiou et Fouchier, 1995; Garcia Sanchez, 2006). Ainsi, les normes de sécurisation, de confort et d'esthétisme ont gagné les espaces de promenades balnéaires.

Par ailleurs, la promenade est un espace de transit entre mer et ville. Elle associe un espace paysager, un espace de rêverie, de régulation des émotions, autrement dit un espace ressourçant (Kaplan, 1995) et un espace construit, minéral, plus fonctionnel et plus normatif également. Face à la ville, la promenade est une « permanence paysagère qui ras- 
sure » (Rieucau, 2009). La présence de l'eau, de la mer, et son effet sur la santé, fait l'objet de divers travaux qui mettent en avant ses bénéfices sur le bien-être (Han, 2007; Wheeler et al., 2012; Völker et Kistemann, 2013). De plus, l'esthétique paysagère, bien souvent centre d'attention des stations balnéaires (Fougnie, 2008), dont les points de vue sont mis en valeur par des aménagements côtiers, en fait des espaces de qualité, voire des «therapeutic landscape »(Gesler, 1993).

\section{Un espace idéal-type pour l'inclusion des personnes âgées?}

Au regard de leur matérialité, les promenades balnéaires respectent les principes de walkability, par ailleurs recherchés par les personnes âgées en ville. Ces caractéristiques en font un lieu de déambulation, de lenteur et de confort. Parce que l'une de ses fonctions originelles est celle de déambuler, la digue de bord de mer ne permettrait-elle pas une malléabilité des pratiques des personnes âgées? C'est-à-dire une possibilité de modifier les pratiques dans cet espace lorsque le vieillissement l'impose? Lorsque les capacités corporelles ne permettent plus de maintenir les distances parcourues et que les difficultés physiques nécessitent de ralentir, voire de se poser, l'espace de la promenade peut-il devenir un espace qui permet de "déprendre " de ses anciennes pratiques en les substituant à d'autres, moins éprouvantes? Dans ce cas, la promenade offrirait un espace d'épanouissement corporel, quelles que soient les capacités physiques et cognitives, à travers les âges. Elle répondrait à la nécessaire mise en œuvre de stratégies ou tactiques d'adaptation des pratiques pour pouvoir garder « prise » : garder prise sur l'espace, sur son corps, mais aussi pour être parmi les autres. Les autres usagers sont en effet bien présents sur les promenades, si l'on en croit les travaux portant sur ces espaces où se confrontent résidents et touristes, et où l'on retrouve la densité et la diversité sociales de la ville. La promenade ne serait-elle pas alors un espace où les individus âgés font pleinement partie du monde et font, voire recherchent, l'expérience de l'imprévu des rencontres? Dans ce sens, la promenade serait à la fois l'espace et la pratique appréciant la compétence citadine des individus, autrement dit la compétence à être en ville, parmi les autres, la compétence à faire la ville dans cet espace symbole, cœur de la station balnéaire. Les pratiques des personnes âgées sur les promenades balnéaires interrogent donc plus largement leur rapport à l'espace urbain, une des dimensions de la citadinité.

Si la promenade est un espace de loisirs pour les retraités, individus au temps libéré, elle est aussi un espace des loisirs pour les pairs : la famille, les amis. De ce fait, l'espace de la promenade interroge les opportunités d'accompagnement et donc celle de faire partie d'un groupe social face aux autres usagers. Entre dépendance et autonomie, le rapport aux pairs semble pouvoir être évolutif sur la promenade.

Enfin, la promenade en tant qu'espace de transition entre mer et ville, peut permettre de questionner le processus de déplacement et de reproduction, sur la promenade, de la centralité des espaces urbains. Au fur et à mesure de l'évolution des rapports des personnes âgées à la ville, la promenade permettrait de maintenir et de contenir des pratiques qui assurent la conservation du sens des lieux. La promenade serait-elle également l'espace qui permet d'assurer une continuité entre les pratiques anciennes et celles qui doivent s'adapter aux effets du vieillissement? Se pose donc la question du rapport au passé dans la citadinité des personnes âgées, l'hypothèse étant que la promenade est un espace de substitution où les personnes âgées peuvent vivre et appréhender les contraintes et les stimulations urbaines à la mesure de leurs compétences et besoins.

\section{Conclusion}

L'inclusion des personnes âgées dans l'espace urbain a ici été traitée à partir de deux notions que sont déprise et citadinité, expliquées et discutées de façon articulée. Nous avons vu que toutes deux permettent de conceptualiser des rapports singuliers aux espaces, dans des lieux où se jouent non seulement la relation à l'autre, mais également les capacités et sentiments d'inclusion à la ville que peut renforcer la relation à l'autre. Les notions de déprise et de citadinité permettent donc de comprendre la notion d'inclusion dans une double perspective : une première temporelle qui sous-tend la notion de déprise et une seconde, plus spatiale, relative à la notion de citadinité. Articuler déprise et citadinité permet de considérer les rapports des personnes 
âgées à la ville comme des transactions, autrement dit un ensemble de pratiques sans cesse renégociées pour maintenir l'équilibre relationnel entre l'individu et la ville. L'individu âgé est en effet pensé dans un processus de vieillissement qui nous amène à considérer ses propres capacités corporelles ainsi que son rapport au monde sous l'effet de la déprise. Ce rapport au monde est étudié avec la notion de citadinité qui met l'accent sur l'inclusion à la ville et au monde.

La mise en place de trois dimensions de la citadinité a servi de cadre à l'analyse de données recueillies dans le cadre d'une thèse de doctorat dont l'exposé des résultats obtenus fera l'objet d'une prochaine publication. Ici, il convenait de poser les bases théoriques de cette recherche afin de nourrir le débat sur l'enjeu de l'inclusion des personnes âgées à la ville.

Au cours de cet article, nous avons voulu montrer l'importance voire la place singulière de certains espaces dans cette question d'inclusion à la ville. Les promenades balnéaires se présentent comme des espaces laboratoires pertinents pour analyser les modalités d'inclusion des personnes âgées. Elles conduisent à nous interroger sur leur disposition à être un espace ressource pour permettre l'ajustement des pratiques grâce à leurs qualités matérielles et sociales, et donc pour répondre aux enjeux de l'inclusion au fur et à mesure que la déprise fait son oeuvre. Parce que l'espace de la promenade favorise la marche, il encourage la présence des personnes âgées et leur permet une place parmi les autres, dans ce haut lieu de la ville.

L'enjeu est alors de penser les promenades balnéaires comme une ressource pour l'avancée en âge, en prenant tout aussi bien en compte leurs aménagements de confort et de sécurisation, que leur attractivité garante d'une densité et d'une diversité sociale.

\section{Bibliographie}

Alves S., 2011. Everyday life in the context of outdoor spaces for older people, Psyecology, vol. 2, n 3, p. 299-307.

Barbedor I., Delignon G., Orain V., Rioult J.-J., 2001. La côte d'Émeraude. La villégiature balnéaire autour de Dinard et Saint-Malo, Paris, Éditions du Patrimoine, 339 p.

Barthe J.-F., Drulhe M., Clément S., 1988. Vieillesse ou vieillissement? Les processus d'organisation des modes de vie chez les personnes âgées, Revue du Centre de Recherches Sur le Travail Social, Caen, $\mathrm{n}^{\circ}$ 15, p. 11-31.

Berry-Chikhaoui I., 2009. Les notions de citadinité et d'urbanité dans l'analyse des villes du Monde arabe, Cahiers d'EMAM, n 18, p. 9-20.

Berry-Chikhaoui I., Deboulet A., 2000. Les compétences des citadins dans le Monde arabe : penser, faire et transformer la ville, Paris, Karthala, 406 p.

Bésingrand D., Soumagne J., 2006. Les séniors mobiles dans les petites villes atlantiques Les exemples de Pornichet et Saint-Jean-de-Luz, Annales de la recherche urbaine, $\mathrm{n}^{\circ}$ 100, p. 83-88.

Bodin F., 2002. Ville et handicap : transporter sans exclure. L'exemple caennais, in Séchet R., Fleuret S. (dir.), La santé, les soins, les territoires: Penser le bien-être, Rennes, PUR, p. 151-165.

Borst H.C., Miedema H.M.E., de Vries S.I., Graham J.M.A., VAn Dongen J.E.F., 2008. Relationships between street characteristics and perceived attractiveness for walking reported by elderly people, Journal of Environmental Psychology, vol. 28, n 4 , p. 353-361.

Bouillon F., Baby-Collin V., Bénit C., Vidal D., 2007. Imprévu, mixité, rencontre, in Gervais-Lambony P. (dir.), Vies Citadines, Paris, Belin, p. 129-148.

Brown B.B., Werner C.M., Amburgey J.W., Szalay C., 2007. Walkable Route Perceptions and Physical Features Converging Evidence for En Route Walking Experiences, Environment Behaviour, vol. 39, n 1, p. 34-61.

Cadiou N., Fouchier V., 1995. La présence végétale dans la ville nouvelle d'Évry. Des usages diversifiés, Annales de la Recherche Urbaine, $\mathrm{n}^{\circ}$ 74, p. 95-102.

Caradec V., 2004. Vieillir après la retraite. Approche sociologique $d u$ vieillissement, Paris, PUF, $240 \mathrm{p}$.

Caradec V., 2005. Les « supports » de l'individu vieillissant. Retour sur la notion de déprise, in Caradec C., Martucelli D., Matériaux Pour Une Sociologie de L'individu : Perspectives et Débats, Arras, Presses Universitaires du Septentrion, p. 25-42.

Caradec V., 2009. L'expérience sociale du vieillissement, Idées Économiques Sociales vol. 157, n³, p. 38-45.

Caradec V., 2012. Épreuve et enjeux du grand âge, in Caradec V., Ertul S., Melchior J.-P. (dir.), Les Dynamiques Des Parcours Sociaux: Temps, Territoires, Professions, Rennes, PUR, p. 91-105.

Carmona M., 1985. Le mobilier urbain, Paris, PUF, 127 p.

Chaudet B., 2004. Une voie « accessible »? Développer des espaces de qualité et de bien-être pour tous, Acte de colloque «Peut-on prétendre à des espaces de qualité et de bien-être? ", Angers, p. 82-85.

Clément S., 1994. La ville et la vieillesse : espace public, temporalité, mobilité, Gérontologie et Société, n 69, p. 150-159.

Clément S., 2003. Le vieillissement avec le temps, et malgré le monde, Empan, n 52, p. 14-22.

Clément S., Mantovani J., Membrado M., 1996. Vivre la ville à la vieillesse : se ménager et se risquer, Annales de la Recherche Urbaine, $\mathrm{n}^{\circ}$ 100, p. 90-98. 
Clément S., Mantovani J., Membrado M., 1998. Expérience du vieillissement et formes urbaines, in Hautmont N. (dir), L'urbain dans tous ses états : faire, vivre et dire la ville, Paris, L'Harmattan, p. 231-243.

Cloutier-Fisher D., Harvey J., 2009. Home beyond the house : Experiences of place in an evolving retirement community., Journal of Environmental Psychology, vol. 29, $n^{\circ} 2$, p. 246-255.

Cluzet A., 2003. Au bonheur des villes, Éditions de l'Aube, $224 \mathrm{p}$.

DeBié F., 1993. Une forme urbaine du premier âge touristique : les promenades littorales. Mappemonde, vol. 1, p. 32-37.

Delignon G., 1998. Urbanisme et architecture balnéaire de Saint-Malo-Paramé : l'invention d'un site (1840-1940). Thèse de doctorat en art et archéologie, université Rennes 2.

Depeau S., 2003. L'enfant en ville : autonomie de déplacement et accessibilité environnementale, thèse de doctorat en psychologie sociale et environnementale, université René-Descartes, $587 \mathrm{p}$.

Dommes A., Cavallo V., Boustelitane F., 2008. La traversée de rue chez le piéton âgé. Effets d'une méthode réentrainement sur simulateur, rapport Inrest/fondation Maif $n^{\circ} 3$.

Drulhe M., Clément S., Mantovani J., Membrado M., 2008. L'expérience du voisinage : propriétés générales et spécificités au cours de la vieillesse, Cahier Internatinal de Sociology, $n^{\circ} 123$, p. 325-339.

Duhamel P., Violier P., 2009. Tourisme et littoral un enjeu du monde, Paris, Belin, 182 p.

Dumont M., Von Der Mühll D., 2006. De la rue à la ville apaisée : l'éclairage comparé des expériences péri/suburbaines suisses et françaises, Flux, vol. 66-67, n 4 , p. 50-61.

Fischer G.N., 1981. La psychosociologie de l'espace, Paris, PUF, $124 \mathrm{p}$.

Fougnie S., 2008. L'intégration géographique comme mode d'interprétation de l'évolution des stations balnéaires, Norois, n² 206, 73-89.

Garcia Sanchez P.J., 2006. Entre urbanité et ordre public. Une écologie de l'usage des places à Caracas, Espace et Sociétés vol. 126, n³, 103-118.

Gervais-Lambony P., 2001. La citadinité, ou comment un mot peut en cacher d'autres..., in Dorier-Apprill E. (dir.), Vocabulaire de La Ville, Paris, Éditions Du Temps, p. $92-108$.

Gesler W. M., 1993. Therapeutic landscapes: theory and a case study of Epidauros, Greece, Environment and planning, vol. 11, n² 2, p. 171-189.

Giddens A., 1987. La Constitution de la société : éléments de la théorie de la structuration, Presses universitaires de France, Paris, $474 \mathrm{p}$.

Guillemot L., Soumagne J., 2007. Temporalités et services en milieu urbain : le cas d'Angers, Espace Population Sociétés, vol. 2 , n³ 3, p. 255-272.

Han K. T., 2007. Responses to Six Major Terrestrial Biomes in Terms of Scenic Beauty, Preference, and Restorativeness, Environment and Behavior vol. 39, $\mathrm{n}^{\circ} 4$, p. 529-556.
Hartig T., StaAts H., 2006. The need for psychological restoration as a determinant of environmental preferences, Journal of Environmental Psychology, vol. 26, n 3, 215-226.

Heam A. S., Dejeammes M., 2000. L'insécurité routière des piétons âgés à travers le système Mobilité - Urbanisme - Réseau, rapport du CERTU. Consulté à l'adresse [http://lara.inist.frl handle/2332/1078].

Hénaff-Pineau P.-C., 2009. Vieillissement et pratiques sportives : entre modération et intensification, Lien Social et Politique, n 62, p. 71-83.

Jaillet M.-C., 1999. Peut-on parler de sécession urbaine à propos des villes européennes?, Esprit, n 253, p. 145-167.

KAPLAN S., 1995. The restorative benefits of nature: Toward an integrative framework, Journal of Environmental Psychology vol. 15, n³, p. 169-182.

KT-EQUAL, 2010. A built environment for all ages, rapport KT-EQUAL.

Lord S., Després C., 2011 . Vieillir en banlieu nord-américaine. Le rapport à la ville des personnes âgées, Gérontologie et Société vol. 1, n³6, p. 189-204.

Lord S., Després C., Ramadier T., 2011. When mobility makes sense: A qualitative and longitudinal study of the daily mobility of the elderly, Journal of Environmental Psychology, vol. 31, n 1 p. 52-61.

Lussault M., 2003. Citadinité, in LÉvy, Lussault (dir.), Dictionnaire de géographie et de l'espace des sociétés, Paris, Belin, p. 159.

Masboungi A., Bourdin A., 2004. Un urbanisme des modes de vie, Le Moniteur, Paris, $96 \mathrm{p}$

Membrado M., 1998. Processus de vieillissement et "secondarité”, in Yerpez J., La Ville Des Vieux, Éditions de l'Aube, p. 95-106.

Membrado M., 2003. Les formes du voisinage à la vieillesse, Empan, n 52, p. 100-106.

Di Méo G., 1998. Géographie sociale et territoires, Paris, Nathan, 320 p.

Michael Y.L., Green M.K., Farquhar S.A., 2006. Neighborhood design and active aging, Health Place, vol. 12, $\mathrm{n}^{\circ} 4$, p. 734-740.

Mollenkopf H., Marcellini F., Ruoppila I., Flaschenträger P., Gagliardi C., Spazzafumo L., 1997. Outdoor mobility and social relationships of elderly people, Archives of Gerontology and Geriatrics, vol. 24, n 3, p. 295-310.

Oliveira A., Abellan A., 1994. Les obstacles physiques de la cité : la brutalité de l'espace construit, Gérontologie et Société, n 69, p. 82-91.

Pennec S., 2005. La pluralité des vieillesses urbaines, in LE Borgne F., Pennec S., Bodin F., Bouchayer F. (dir.), Technologies Urbaines, Vieillissements et Handicaps, Rennes, Presses de EHESP, p. 153-169.

Pennec S., 2006. Les pratiques de la ville entre anonymat et proximité Garder une relation urbaine au monde, Annales de la Recherche Urbaine, $\mathrm{n}^{\circ}$ 100, p. 51-58.

Pinet C., 2006. Du vieillissement dans les villes à des villes pour le vieillissement? Réflexions sur quelques exemples français, Annales de la Recherche Urbaine, n 100, p. 15-22. 
Ramirez B., K, L., Hoehner C.M., Brownson R.C., Cook R., Orleans C.T., Hollander M., Barker D.C., Bors P., Ewing R. et al., 2006. Indicators of Activity-Friendly Communities: An Evidence-Based Consensus Process, American Journal of Preventive Medicine, vol. 31, n 6, p. 515-524.

Ridolfi G., Dumont I., 2006. Promenades pour l'âge d'or dans deux petites villes italiennes, Annales de la Recherche Urbaine, $\mathrm{n}^{\circ}$ 100, p. 97-105.

Rieucau J., 2009. Vers des plages urbaines postbalnéaires au début du Xxi ${ }^{\mathrm{e}}$ siècle, Géographie et Cultures, nº 67, p. 27-46.

Rouillard D., 1984. Le site balnéaire, Bruxelles, Mardaga, $357 \mathrm{p}$.

Saelens B., Sallis J., Frank L., 2003. Environmental correlates of walking and cycling: Findings from the transportation, urban design, and planning literatures, Annals of Behavioral Medicine, vol. 25, n² 2, p. 80-91.

Séchet R., Garat I., Zeneidi D. 2008. Introduction, in Séchet R., Garat I., Zeneidi D., Espaces en transactions, Rennes, PUR, p. 7-26.
Touboul P., Valbousquet J., Pourrat-Vanoni I., Alquier M.-F., Benchimol D., Pradier C., 2011 . Comment adapter l'environnement pour favoriser la marche des seniors? Une étude qualitative, Santé Publique, vol. 23, p. 385-399.

Völker S., Kistemann T., 2013. "I'm always entirely happy when I'm here!" Urban blue enhancing human health and well-being in Cologne and Düsseldorf, Germany, Social Science E Medicine, vol. 78, p. 113-124.

Von Der Muhll D. (2004). Mobilité douce : nostalgie passéiste ou perspective d'avenir, in Vodoz L., Pfister B., Jemelin C. (dir.), Les territoires de la mobilité. L'aire du temps, Presses polytechniques et universitaires romandes, p. 209-224.

Wheeler B., White M., Stahl-Timmins W., Depledge M., 2012. Does living by the coast improve health and wellbeing?, Health and Place, vol. 18, n 5, p. 1198-1201. 